\title{
Erratum: Heterogeneity in synaptic transmission along a Drosophila larval motor axon
}

Giovanna Guerrero, Dierk F Rieff, Gautam Agarwal, Robin W Ball, Alexander Borst, Corey S Goodman \& Ehud Y Isacoff Nat. Neurosci., 8, 1188-1196 (2005)

In the version of this article initially published online, the second author's name was misspelled. The correct spelling is Dierk F Reiff.

\section{Erratum: Notch signaling in the mammalian central nervous system: insights from mouse mutants}

\author{
Keejung Yoon \& Nicholas Gaiano \\ Nat. Neurosci., 8, 709- $715(2005)$
}

The version of this article that was published contained typographical errors in some gene names. On page 710, in the right column, third paragraph, the fourth sentence should have read as follows: "That study examined the expression both of pathway components such as Hes1, Hes5 and Delta-like 1 (Dll1) and of early differentiation markers such as Math4A (also known as Neurog2), NeuroD and NSCL-1 (also known as Nhlh1)." The last sentence of that paragraph should have read as follows: "This notion is supported by previous findings that Hes1 can be upregulated in PC12 cells cultured in the growth factors NGF, FGF2 or EGF64 and in postnatal cerebellar granule cells cultured in Sonic hedgehog 65 ." The fourth and fifth sentences in the second paragraph, right column, on page 713 should have read as follows: "These studies found that Notch activation reduced neurite extension, but presumed signaling blockade (via expression of Numb, Numbl or Dx) could promote neurite extension. Subsequent studies have found that Numb deletion disrupts neuronal maturation in the developing cerebellum ${ }^{31}$, whereas deletion of Numb and Numbl disrupts axonal arborization in sensory ganglia in vivo ${ }^{32}$." In addition, on page 712 , in the right column, top line, the authors would like to revise the sentence to read as follows: "Third, several reports have identified ErbB2 as a Notch target that has a role during mammalian radial glial maintenance 91,92 ."

COR RIGENDUM

Corrigendum: Visual field maps and stimulus selectivity in human ventral occipital cortex

Alyssa A Brewer, Junjie Liu, Alex R Wade \& Brian A Wandell

Nat. Neurosci., 8, 1102-1109 (2005)

The discussion section contains an incorrect citation. In the 3rd paragraph on page 1107, “Tootell et al. ${ }^{16}$ (subsequent to Halgren et al.)" should read: "Tootell et al. ${ }^{16}$ (subsequent to Hadjikhani et al.)". The authors regret the error 